\title{
The effect of different recovery methods on muscle swelling recovery after muscle fatigue
}

\author{
Fan Zhang 1, 2, a , Zhuying Wang 2, b \\ 1Department of Police Skills and Tactics, Nanjing Forest Police College, Nanjing 210023, China; \\ ${ }^{2}$ Sports Science postdoctoral programme, Nanjing Normal University, Nanjing 210023, China. \\ azhangfan@nfpc.edu.cn, bwangzhuying@njnu.edu.cn
}

Keywords: muscle swelling; muscle fatigue; centrifugal motion; vibration recovery; cryotherapy. L manuscripts

\begin{abstract}
This paper's purpose is to investigate the effect of passive recovery (PR), active recovery (AR), vibration recovery (VR) and cryotherapy recovery (CR) on muscle swelling Degree (SD) after muscle fatigue. 20 healthy male college students were enrolled in the study. SD of the biceps brachii of the non-handedness was measured. The differences and recovery effects of SD at different detection time points were tested by one-way repeated measures ANOVA. Results shows that Continuous AR of decreasing intensity cannot improve recovery of fatigue muscle and even increase SD after active concentric contraction and passive pulling at constant speed in low intensity of $45 \%$ s. In addition, CR can effectively control tissue injury and SD problem due to centrifugal motion.
\end{abstract}

\section{Introduction}

Muscle fatigue sourced from continuous muscle contraction will lower late exercise performance and bring muscle injury response, and therefore, if effective recovery method is used within limited time, it will be quite beneficial to re-performance improvement and exercise injury reduction. Among them, muscle swelling is an important index to measure the effect of muscle recovery.In previous research; it is found that if mild concentric contraction is implemented under muscle injury, elimination of interstitial fluid accumulation within tumid muscle will be quickened [1], thus improving recovery efficiency [2]. Vibration training rising recently belongs to passive recovery method that can generate effect similar with active recovery without active muscle contraction, and this characteristics are quite helpful to fatigue recovery. In addition, cryotherapy recovery can also be adopted to lower muscular temperature and promote vasoconstriction, thus lowering blood flow volume, inflammation, tumidness and ache conditions of injured muscle. But no matter what kind of recovery effect may vary with method and time limit [3], and therefore final conclusion does not appear.

\section{Research object and method}

\subsection{Object of study}

20 male undergraduates having healthy body are subjects (they are free from cardiovascular disease, high blood pressure and relevant viscera disease, and they can receive the maximum exercise test), with average age $(20.4 \pm 2.3 \mathrm{yrs}$.), average height $(173.5 \pm 3.4 \mathrm{~cm})$ and average weight $(68.7 \pm 3.9 \mathrm{~kg})$.

\subsection{Experimental design.}

Experimental design of repetitive measurement is adopted, and every subject accepts four different fatigue recovery ways in anti-balance sequence method: PR, AR, VR, CR. Interval of experimental procedure in every step shall be over 7 days to avoid that delayed onset muscle soreness disturbs experimental result.

1) In phase of familiarization with actions, elbow joint shall bend to contract 10 times centripetally and eccentrically repeatedly in $45^{\circ}$ s of angular speed;

2) In phase of measurement before fatigue, BBM Swelling Degree (SD) shall be measured in sequence to avoid that mechanical property is affected by muscle manifestation test. 
3) In phase of muscle fatigue, isokinetic exercise system of Biodex System Pro-4 is adopted, and maximum contraction exercise shall be conducted centripetally and eccentrically repeatedly in $45 \%$ of angular speed on bent of non-habitual elbow joint, and activity scope is $30 \sim 120^{\circ}$ (it is $0^{\circ}$ when elbow completely stretches straightly), and muscles can be judged in fatigue until finally there are continual three times where muscle force manifestation reduces to $50 \% \mathrm{MVC}$, and then exercise of constant speed can be stopped.

4) In phase of measurement after fatigue, data of muscle SD shall be immediately measured.

5) In phase of intervening of recovery way, one of its recovery ways can be conducted in random way: PR group shall rest for 10 minutes in still sitting gesture; concentric contraction exercise shall be conducted for AR group in constant speed on isokinetic exercise system of Biodex System Pro-4. It shall start in strength of $25 \% \mathrm{MVC}$, and the speed shall decrease by $5 \%$ MVC every two minutes, and there shall be 10 minutes totally. For VR group, vibration training platform Power Plate Pro5 AIR ${ }^{\mathrm{TM}}$ shall be used to partially vibrate BBM, and vibration frequency shall be $30 \mathrm{~Hz}$, amplitude being $2 \mathrm{~mm}$ for 10 minutes. Ice compression shall be conducted on fatigue parts with LP ice pack (LP894; M:22.9 $\mathrm{cm} ; 65 \%$ ice block filling) non-pressure device for 10 minutes for CR group.

6) In phase of measurement after recovery, SD of BBM shall be measured immediately.

7) Then experiment of another recovery way shall be conducted until totally recovery with interval over 7 days.

\subsection{Test data and collection.}

1) Muscle swelling Degree. Biceps circumference can be measured with a tape, and the tape can surround BBM muscle belly. The maximum biceps circumference $(\mathrm{cm})$ shall represent SD degree.

2) Recovery effect. Values of SD at different measurement time points (before fatigue, after fatigue and after recovery) shall be calculated, and then [(after recovery/before fatigue) $\times 100 \%$-(after fatigue/before fatigue) $\times 100 \%$ ] shall be deemed as recovery effect of SD.

\subsection{Statistical analysis.}

Statistical handling shall be conducted via SPSS 19.0, and the result shall be represented with mean value \pm standard deviation $(\bar{X} \pm \mathrm{SD})$. Difference of SD in all groups (PR, AR, VR, CR) at different time points (before fatigue, after fatigue, after recovery) shall be compared respectively via repeated measures of a single factor. Difference among groups of SD can also be compared, and posterior comparison can be conducted via way of Bonferroni if standard reaching is significant. Statistical significance level is $P<0.05$, and non-significance level is $P<0.01$.

\section{Change of Swelling Degree after intervening of different recovery ways}

\subsection{Difference of different time points.}

SD in all groups reaches significant difference at different time points $(P<0.05)$. MSL in all groups after fatigue is significantly more than that before fatigue $(P<0.05)$ after posterior comparison, while only SD in CR group after recovery is significantly less than that after fatigue $(P<0.05)$ (Table 1$)$.

Table 1 . SD of different recovery methods at different time points

\begin{tabular}{|c|c|c|c|}
\hline $\mathrm{SD}(\mathrm{cm})$ & before fatigue & after fatigue & after recovery \\
\hline PR & $27.69 \pm 2.52$ & $28.22 \pm 2.53^{\mathrm{a}}$ & $28.05 \pm 2.49^{\mathrm{b}}$ \\
\hline AR & $27.93 \pm 2.93$ & $28.37 \pm 2.88^{\mathrm{a}}$ & $28.51 \pm 2.79^{\mathrm{b}}$ \\
\hline VR & $28.02 \pm 2.91$ & $28.44 \pm 2.83^{\mathrm{a}}$ & $28.24 \pm 2.79$ \\
\hline CR & $27.80 \pm 2.43$ & $28.21 \pm 2.28^{\mathrm{a}}$ & $28.01 \pm 2.29$ \\
\hline
\end{tabular}

Note:a represents significant difference before fatigue and after fatigue; $b$ represents significant difference before fatigue and after recovery. $P<0.05$. 


\subsection{Difference of recovery effect.}

Recovery effects of all groups reach significant difference $(P<0.05)$ (Fig. 1); SD after recovery of $\operatorname{AR}(0.53 \pm 0.99 \%)$ is significantly more than that of $\operatorname{PR}(-0.61 \pm 0.83 \%)$ and $\operatorname{CR}(-0.76 \pm 0.89 \%)(P$ $<0.05)$, while there is no significant difference $(P>0.05)$ between VR $(-0.7 \pm 1.43 \%)$ and other groups.

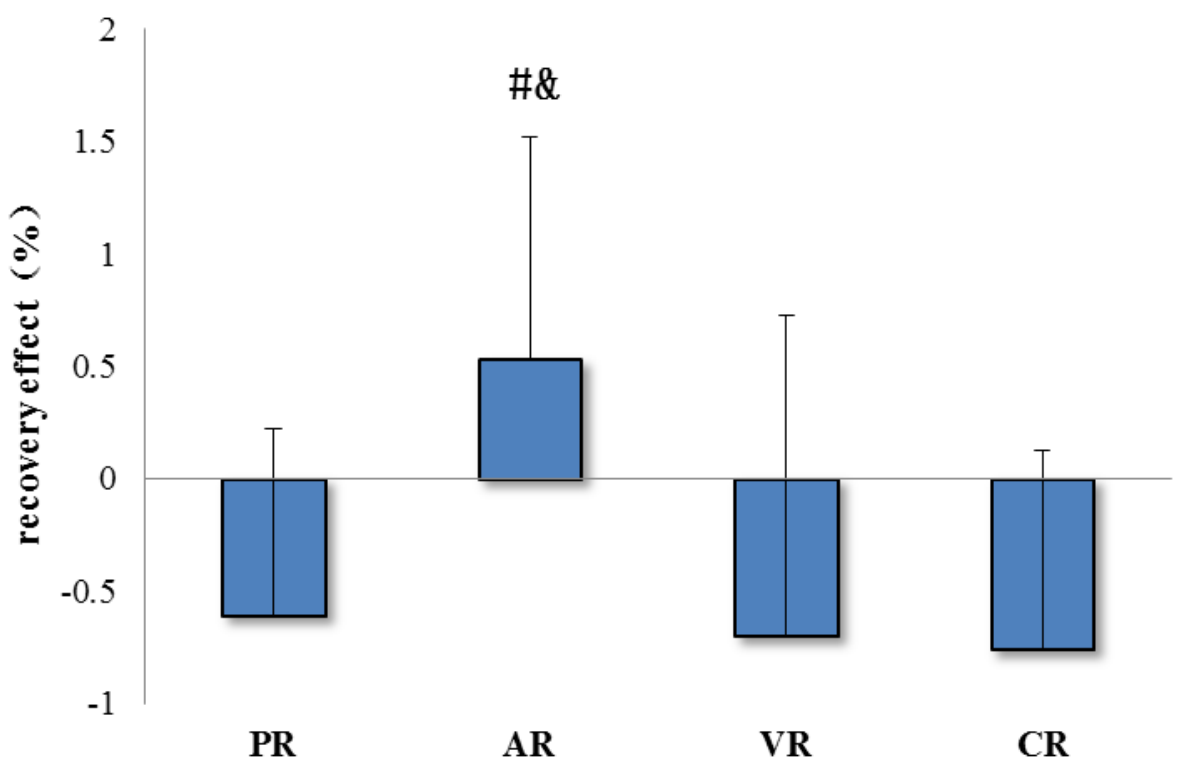

Fig.1 Comparison of the recovery effect of SD

Note: \# represents significantly better than group PR; \& represents was better than that of group CR. $\mathrm{P}<0.05$

\section{Summary}

After plenty of muscle exercise, phenomena such as fatigue, injury, etc will appear. Especially muscle injury can be more easily caused after centrifugal exercise, which will lead to liquid containing protein permeating and piling in muscle fiber and then producing SD[4]. It is indicated in researches that phenomenon that there is obvious SD in upper arm is indeed caused after fatiguing exercise, and it is consistent with the opinion proposed in previous researches[5] that fatiguing exercise causes muscle injury and then promote swelling of bicep circumference. So these debris causing muscle swelling shall be stopped to produce and eliminated speedily, so as to avoid imposing adverse influence on exercise performance and feeling of comfort due to hurt from muscle swelling.

It is indicated in experimental results that CR can obviously relieve SD, and relieve effect of AR is worse than PR and CR. It is pointed out in previous researches[6] that partial CR can reduce the temperature of partial tissues, promote micrangium contraction, exert function of hemostasis and detumescence, increase blood viscocity, reduce blood flow volume for wounded tissues. It can also reduce enzyme activity of metabolism and correspondingly reduce tissue metabolism and reduce oxygen demand volume (to avoid affecting other unwounded tissues due to lack of oxygen), then it can further reduces injury and lower SD. Though it is recommended in previous researches that CR duration shall be 15 20 minutes, only 10 minutes of ice compression is conducted on limb in this research. But there are scholars pointing out direct ice compression on muscle belly of right leg for 10 minutes not adding pressure can obviously lower temperature of partial tissues. For muscle group in this research is BBM belonging to small muscle group, then it is assumed that partial CR can effectively control SD, and it has been proved in the experiment.

Besides, PR, AR, VR all do not reach obvious recovery effect, even average value of bicep circumference increases after AR recovery. It is indicated that AR recovery not only cannot slower SD, but it worsens the situation. Though it is indicated in researches that AR recovery can boost blood circulation[7], then the reason why it is not consistent with this research may be that continual active contraction after muscle injury cause ineffective rest for muscles of fatigue injury. It causes piling of 
interstitial fluid over that eliminated, so the body cannot obtain fatigue recovery and negative effect is caused.

\section{Acknowledgments}

This work was supported in part by the Project of the Fundamental Research Funds for the Central Universities under Grant LGZD201709, in part by the Project of China Postdoctoral Science Foundation under Grant 2017M611849, in part by Jiangsu Qing LAN Project under Grant 2017, and in part by Nanjing Forest police College Teaching Reform Project under Grant ZD17001 \& YB17001.

\section{References}

[1]. Mohr T, Akers T K, Wessman H C. Effect of high voltage stimulation on blood flow in the rat hind limb. Physical Therapy. Vol. 67 (1987) No. 4, p.526-533.

[2]. Clemente F R, Matulionis D H, Barron K W, et al. Effect of motor neuromuscular electrical stimulation on microvascular perfusion of stimulated rat skeletal muscle.[J]. Physical Therapy .Vol. 71 (1991) No. 5, p.397-404.

[3]. ZHANG F, WANG C S, YE Z Q, et al. An Experimental Study Comparing the Effect of Different Stretching Mode on Hamstrings Flexibility. Journal of Tianjin University of Sport. Vol. 29 (2014) No. 1, p. 61-65. (In Chinese)

[4]. Proske U, Morgan D L. Muscle damage from eccentric exercise: mechanism, mechanical signs, adaptation and clinical applications. Journal of Physiology. Vol. 537 (2001) No. 2, p.333-345.

[5]. Niitsu M, Michizaki A, Endo A, et al. Muscle hardness measurement by using ultrasound elastography: a feasibility study .Acta Radiologica .Vol. 52 (2011) No. 1, p.99-106.

[6]. Swenson C, Swärd L, Karlsson J. Cryotherapy in sports medicine. Scandinavian Journal of Medicine \& Science in Sports. Vol. 6 (1996) No. 4, p.193-200.

[7]. Osada T, Saltin B, Göran R. Assessment of voluntary rhythmic muscle contraction-induced exercising blood flow variability measured by Doppler ultrasound [J]. Open Journal of Molecular \& Integrative Physiology. Vol. 3 (2013) No. 4, p.158-165. 